\title{
Structural Characterization of Endoribonuclease Nsp15 from SARS CoV-2
}

\author{
Youngchang Kim ${ }^{1}$, Natalia Maltseva ${ }^{1}$, Changsoo Chang ${ }^{1}$, Mateusz Wilamowski ${ }^{2}$, Robert Jedrzejczak ${ }^{1}$, \\ Jacek Wower ${ }^{3}$, Glenn Randall' ${ }^{2}$ Karolina Michalska' ${ }^{1}$, Andrzej Joachimiak ${ }^{1,2}$ \\ ${ }^{1}$ Argonne National Laboratory, Lemont, United States of America; \\ ${ }^{2}$ University of Chicago, Chicago, United States of America; \\ ${ }^{3}$ Auburn Univeristy, Auburn, United States of America \\ ykim@anl.gov
}

In response to emergence of global COVID-19 pandemic, studies of SARS-CoV-2 have been well underway with an unprecedentedly fast phase particularly for vaccine development. While spike proteins and proteases Mpro and PLpro are getting much of attention as therapeutic drug targets against COVID-19, however, the progress in developing drugs is still lagging behind. Non-structural protein 15 (Nsp15) is another SARS-CoV-2 protein demanding researchers' attention as a critical drug target. Nsp15 is an endoribonuclease and an essential enzyme for SARS CoV-2 with a role of interfering host immune response. It has been reported that Nsp15 is evading melanoma differentiation-associated gene 5 (MDA5) activity which is triggered by ds/ssRNA molecular pattern produced by replication-transcription complex by trimming replication produced (-) strand polyU track. We characterized Nsp15 by crystallography, biochemical, and whole-cell assays. Several structures of Nsp15: the Apo-form and several ligands bound forms are determined. Our Nsp15 structures with nucleotide-base ligands elucidated how Nsp15 recognizes uridine base specifically. The structure with a transition state analog, uridine vanadate, confirms interactions key to catalytic mechanisms which is mimicking that of RNaseA. We also found an uracil analog Tipiracil, an FDA approved drug that is used in the treatment of colorectal cancer, as a potential anti-COVID-19 drug. These findings can be new insights for the development of uracil scaffold-based therapeutic drugs.

Keywords: Nsp15, endoribonuclease, SARS CoV-2 